\title{
Modeling of Thermoacoustic Frequency Characteristics for the Air-Tightness Test Method
}

\author{
M. Kubicki • M. Maliński
}

Received: 24 October 2013 / Accepted: 11 June 2014 / Published online: 17 July 2014 (C) The Author(s) 2014. This article is published with open access at Springerlink.com

\begin{abstract}
This paper is a description of theoretical models applied for the numerical interpretation of the experimental amplitude and phase frequency thermoacoustic characteristics observed for the transistor's packaging TO-39 with model holes of known radii in the packaging. They are essential from the point of view of determination of the sensitivity of the thermoacoustic method applied for the measurements of the air-tightness of packagings of electronic elements. In this paper, two models of the thermoacoustic signal are described, which are based on a transformation of the thermoacoustic effect to an electrical circuit: CRC and CRLC. Additionally, a method of extraction of the signal, coming from the hole in the packaging, from the total thermoacoustic signal, of the TO-39 packaging, is presented and discussed. The limitation of this method of detection of the air-tightness, caused by the thermoacoustic signal of the air-tight packaging, is also discussed.
\end{abstract}

Keywords Hermeticity testing - Non-destructive testing - Thermoacoustic test method

\section{Introduction}

The hermeticity of electronic components is a crucial reliability parameter, especially under extreme conditions (high temperature, humidity, or pressure) or when the high reliability of electronic devices is essential (e.g., avionics, medicine). Standards [1-5] define test methods which are used in the electronics industry. The main disadvantage of a majority of the methods included in the industrial standards is their destructivity,

M. Kubicki $(\varangle) \cdot$ M. Maliński

Department of Electronics and Computer Science, Koszalin University of Technology, 2 Śniadeckich St, 75453 Koszalin, Poland

e-mail: kubicki.maciej@gmail.com 
which limits their use only to the statistical approach. Many authors propose alternative solutions for the methods used in the industry [6-13]. It is possible to divide those methods into two main groups. The first group of methods uses different types of spectroscopy techniques: Fourier transform infrared spectroscopy [6-8], Raman spectroscopy [8], and near-infrared spectroscopy [9]. The second group of methods described in [10-13] uses different piezoelectric, resistive, or capacitive internal structures of the component which monitor the changes of the internal pressure or the humidity increase inside of the package. The thermoacoustic test method presented in this article may be considered as an another example of a non-destructive alternative for destructive industrial hermeticity tests, especially for destructive gross leak detection methods such as a fluorocarbon bubble test or dye penetrant liquid test. The method presented in the article is intrinsically non-destructive, as it does not use any agents which may penetrate the package of the component and break its integrity. The basic principles of the considered method and example results have been shown in previous papers [14-16]. The current paper focuses on modeling of different phenomena linked with the package air-tightness and factors which limit the sensitivity of the method.

\section{Description of the Method}

The mechanism of the measurement used in the thermoacoustic test method lies in a controlled heat dissipation in the tested component. During the test, the measured device (e.g., bipolar transistor) is closed in the acoustic chamber and a periodical Joule's heat is dissipated in the component's chip driven by an external control signal. A schematic of the basic test system is shown in Fig. 1. In case of leakiness in the component's package, there will be an air flow through the leak channel of radius $r$ and length $l$ causing periodical overpressure changes inside the test chamber. These changes can be detected with a test microphone $M$, whose output signal, after amplification in a preamplifier circuit $K$ and further processing (e.g., detection with a lock-in amplifier), allows estimation of the size of the leak. For each particular case, the amplitude and phase thermoacoustic characteristics can be obtained in the whole acoustic spectrum. As was shown in previous studies [14-16], the shape of those characteristics is dependent on the dimensions of the leakiness and allows for the estimation of its size. For the purposes of the evaluation of the sensitivity of the thermoacoustic method

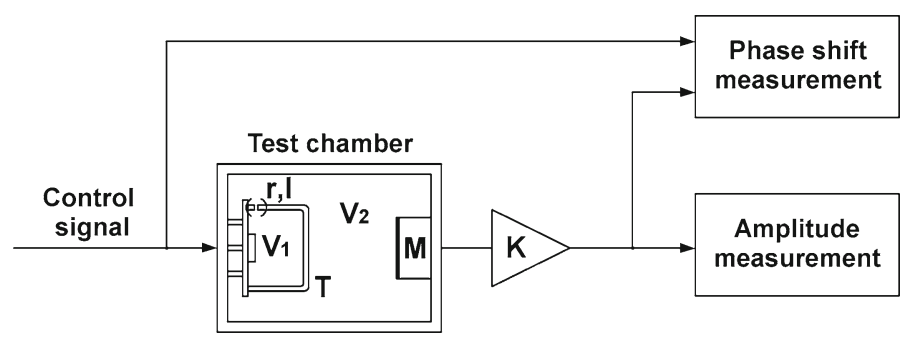

Fig. 1 Idea of the thermoacoustic hermeticity test method 


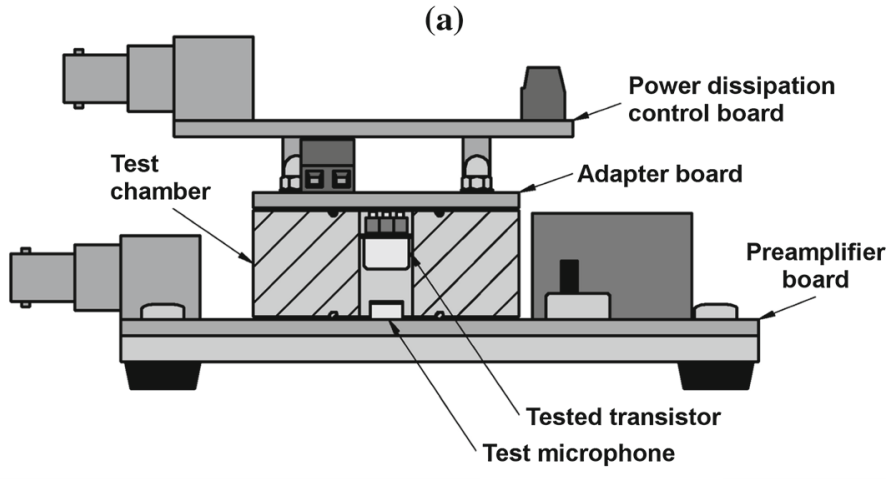

(b)

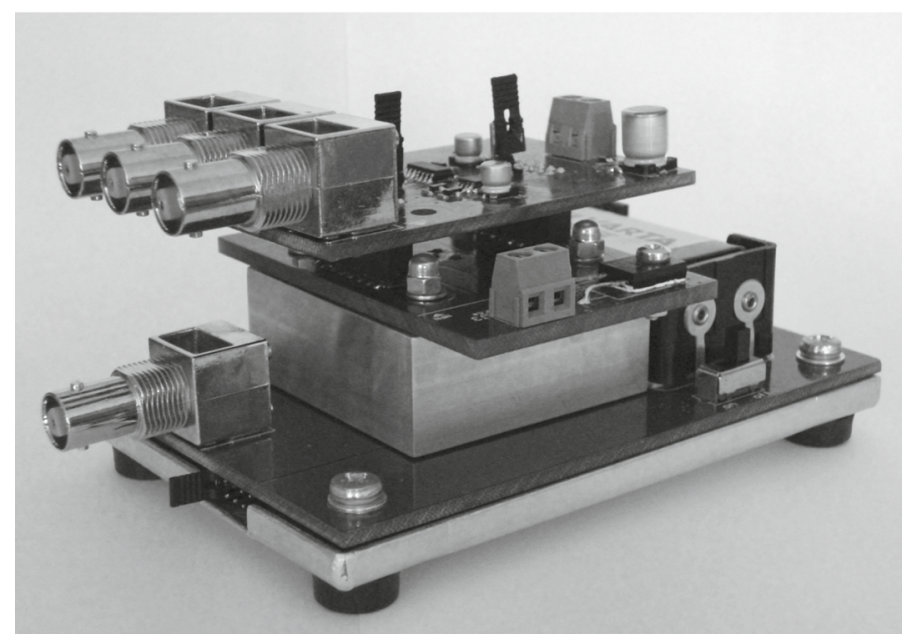

Fig. 2 (a) Schematic of the thermoacoustic chamber setup and (b) photograph of the thermoacoustic chamber setup

and comparison of the mathematical models, the experimental setup was built. The main part of the setup is the acoustic test chamber coupled with the battery-powered preamplifier board integrated with the test microphone and the circuitry for driving the tested component. The cross-section of the test chamber setup is shown in Fig. 2a. The photograph of the acoustic chamber setup used in the experiments is presented in Fig. 2b.

The presented experimental setup was used in a connection with the data acquisition card and measurement software designed in the LabView environment. The main function of the measuring software is generation of the control signal for driving the tested component and the measurement of the phase and amplitude of the output signal coming from the preamplifier board. For the purpose of the measurement of low signals in a noisy environment, the measurement software implements a well-known lock-in detection algorithm. 


\section{Comparison of the Mathematical Models}

The selection of an appropriate mathematical model is essential from the point of view of a further interpretation of the measurement data. The considered models are CRC and CRLC. Both models are based on a transformation of the thermoacoustic effect to an electrical circuit. The idea of the transformation was shown in previous works [14-17].

\subsection{CRC Basic Model}

The idea of the CRC model was presented in previous papers [14-16]. The schematic diagram of a considered electrical model is shown in Fig. 3a. The volumes $V_{i}$ of the test chamber $(i=1)$ and the tested component $(i=2)$ are represented by electrical capacitances $C_{i}$ given by

$$
C_{i}=\frac{M V_{i}}{N_{\mathrm{A}} k T}
$$

where $M$ and $T$ are, respectively, the molar mass of gas (air) and the temperature, $N_{\mathrm{A}}$ is the Avogadro number, and $k$ is the Boltzmann constant.

The flow of the gas through the leakiness of radius $r$ and length $l$ is represented by an electrical resistance given by

$$
R=\frac{8 \eta l}{\rho \pi r^{4}}
$$

where $\eta$ and $\rho$ are, respectively, the viscosity and density of air.

The output signal can be described by the following equation:

$$
U_{\mathrm{o}}(\omega)=\left[\frac{1}{\mathrm{j} \omega\left(C_{1}+C_{2}\right)-\omega^{2} C_{1} C_{2} R}\right] I(\omega),
$$

Fig. 3 (a) CRC electrical model, where $C$ is the capacitance and $R$ is a resistance and (b) CRCL electrical model, where $C$ is the capacitance, $R$ is a resistance, and $L$ is the inductance (a)

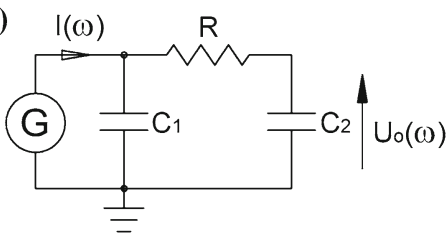

(b)

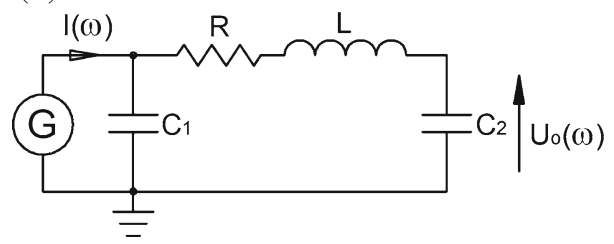


where the current source $I(\omega)$ represents the driving force for the heat dissipation in the component's chip.

For a circuit presented on the schematic from Fig. 3a, we can consider a special case when the resistance $R=0$. This case represents a situation when the component's package is fully opened. Substituting $R=0$, Eq. 3 simplifies to the form,

$$
U_{\mathrm{o}}(\omega)_{R=0}=\left[\frac{1}{\mathrm{j} \omega\left(C_{1}+C_{2}\right)}\right] I(\omega) \text {. }
$$

By dividing Eq. 4 by Eq. 3, we get a transmittance representing the thermoacoustic characteristic of the tested component,

$$
\frac{U_{\mathrm{o}}(\omega)}{U_{\mathrm{o}}(\omega)_{R=0}}=\frac{\omega\left(C_{1}+C_{2}\right)}{\omega\left(C_{1}+C_{2}\right)+\mathrm{j} \omega^{2} C_{1} C_{2} R} .
$$

Transmittance (Eq. 5) can be obtained experimentally by measuring the signal of the fully opened package and the package with the potential leakiness. Measurement of the reference fully opened package frees the results from the frequency characteristics of the measuring equipment and the absolute value of the power dissipated in the component. By fitting the theoretical curves given by Eq. 5 to the experimental data, it is possible to estimate the size of the leakiness.

\subsection{CRLC Extended Model}

The idea of the CRLC model was presented in [17]. The basic model is extended by adding an electrical inductance as it is shown in Fig. 3b. From [17], the value of the inductance can be described by the equation,

$$
L=\frac{\rho l}{\pi r^{2}}
$$

By using the same methodology as previously, the transmittance representing the thermoacoustic characteristic of the tested component can be described by the equation,

$$
\frac{U_{\mathrm{o}}(\omega)}{U_{\mathrm{o}}(\omega)_{R=0, L=0}}=\frac{\omega\left(C_{1}+C_{2}\right)}{\omega\left(C_{1}+C_{2}\right)+\mathrm{j} \omega^{2} C_{1} C_{2}(R+j \omega L)} .
$$

\subsection{Comparison of the Models' Accuracy}

For the purpose of comparison, a series of measurements of NPN bipolar transistors in TO-39 packages of known leakiness sizes were performed and subsequently theoretical fittings to the measurement data were done using both models. Figure 4 shows the obtained phase and amplitude thermoacoustic characteristics together with the theoretical fitting for different sizes of the package leakiness. In the plots of Fig. 4, it can be 

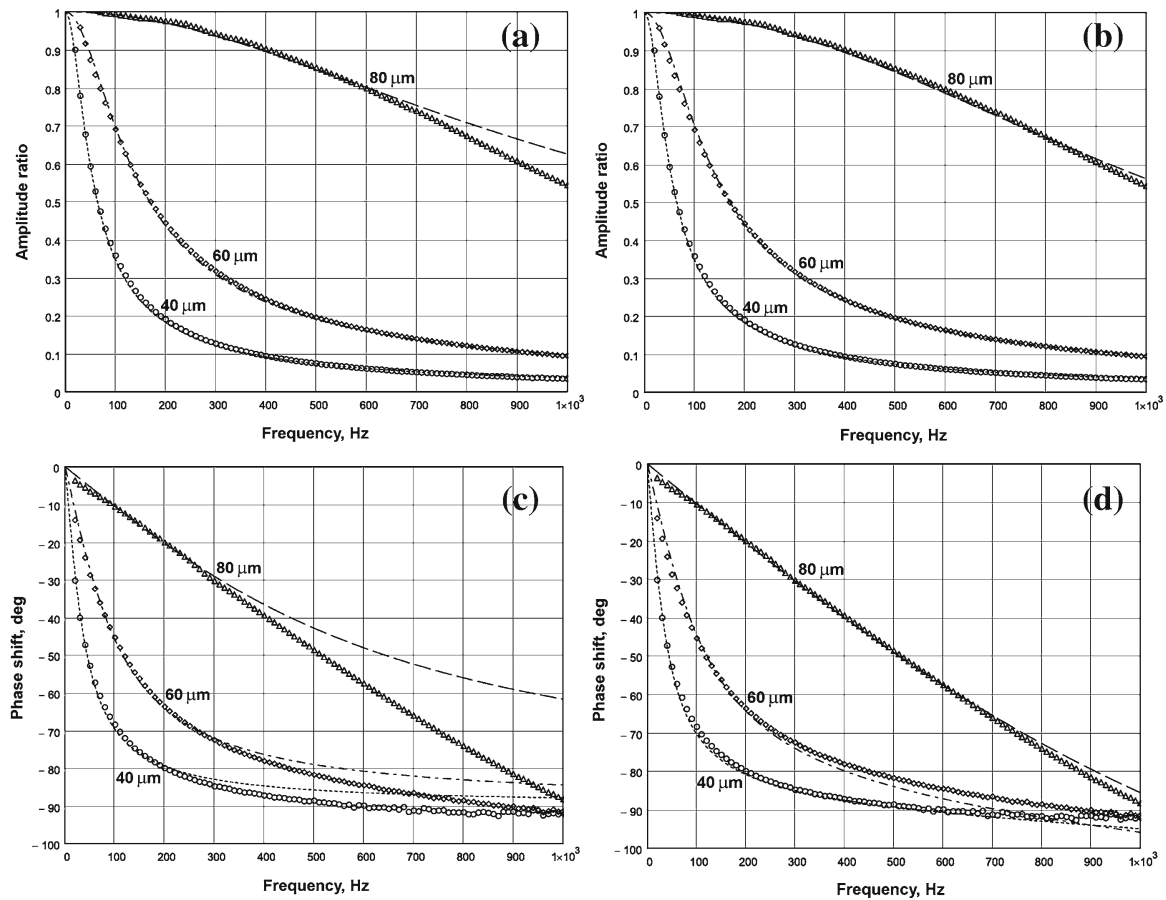

Fig. 4 Thermoacoustic frequency characteristics of TO-39 packages with leakinesses of different radii together with theoretical fittings: (a) amplitude characteristics experimental data and CRC model theoretical fittings, (b) amplitude characteristics experimental data and CRLC model theoretical fittings, (c) phase characteristics experimental data and CRC model theoretical fittings, and (d) phase characteristics experimental data and CRLC model theoretical fittings

seen that both models fit well for low frequencies. For high frequencies, discrepancies between the theoretical curves of both models and the experimental data are observed. However, the CRLC extended model yields a better fit to the experimental data than does the CRC model.

\section{Sensitivity Limiting Factors}

There are three processes that exist when the packaging is not air-tight. The first process is when the chip heats up the packaging. It next heats up the air in the acoustic chamber which results in periodical changes of the overpressure in the chamber. The second process is when the gas from the transistor flows periodically, through the hole, to the acoustic chamber, and causes the additional periodical changes of the overpressure in the acoustic chamber. The third process is when the temperature gradient in the base of the package bends it periodically causing a drum effect. Apart from a limited sensitivity of the measuring equipment (resolution of data acquisition card) and noise conditions (acoustic noise in the chamber, thermal noise in a preamplifier circuitry, electromagnetic noise in the test environment), the main limiting factor is the signal 
coming from the air-tight package of the component. It is called a background signal. There are two main mechanisms of a background signal generation. The first one is the heat dissipation in the component's chip and diffusion of the thermal wave through the semiconductor structure and the metal base of the package to the acoustic chamber. The second one is the drum effect in the metal base of the component. Both mechanisms were modelled, and the models were compared with the experimental data.

\subsection{Thermal Wave in the Component's Package}

The thermal wave component of the acoustic background signal can be modelled with the two-layer model presented in [18] considering the semiconductor layer and the metal base of the device as the second layer. The pressure signal seen in the front (on the surface of the semiconductor chip) can be described by the formula,

$$
P_{\mathrm{F}}(f)=\frac{I(f)\left(\cosh \left(l_{1} \sigma_{1}(f)+l_{2} \sigma_{2}(f)\right)+R_{12} \cosh \left(l_{1} \sigma_{1}(f)-l_{2} \sigma_{2}(f)\right)\right)}{\lambda_{1} \sigma_{1}^{2}(f)\left(\sinh \left(l_{1} \sigma_{1}(f)+l_{2} \sigma_{2}(f)\right)+R_{12} \sinh \left(l_{1} \sigma_{1}(f)-l_{2} \sigma_{2}(f)\right)\right)} .
$$

The signal seen in the reverse (outside of the component) is described by the formula,

$$
P_{\mathrm{R}}(f)=\frac{I(f)\left(1+R_{12}\right)}{\lambda_{1} \sigma_{1}^{2}(f)\left(\sinh \left(l_{1} \sigma_{1}(f)+l_{2} \sigma_{2}(f)\right)+R_{12} \sinh \left(l_{1} \sigma_{1}(f)-l_{2} \sigma_{2}(f)\right)\right)} .
$$

In the above equations, $\lambda_{1}$ is the thermal conductivity of the semiconductor layer, and $l_{1}, l_{2}$ are the thicknesses of the semiconductor and metal base layers, respectively. $I(f)$ represents the driving signal for the heat dissipation.

$R_{12}$ is a function of the thermal effusivities of the layers given by the equation,

$$
R_{12}=\frac{e_{1}-e_{2}}{e_{1}+e_{2}}
$$

$\sigma_{i}$ ( $i=1$ for the semiconductor layer, $i=2$ for the metal base layer) are functions of the thermal diffusivities $\alpha_{i}$ of the layers and the frequency of the driving signal:

$$
\sigma_{i}(f)=\frac{1+\mathrm{j}}{\sqrt{\frac{\alpha_{i}}{\pi f}}} .
$$

The relations between the reverse signal and the front signal are described by the formulae:

$$
\begin{aligned}
A_{\mathrm{R} 2 \mathrm{~F}} & =\left|\frac{P_{\mathrm{R}}(f)}{P_{\mathrm{F}}(f)}\right|, \\
\varphi_{\mathrm{R} 2 \mathrm{~F}} & =\frac{180}{\pi} \arg \left(\frac{P_{\mathrm{R}}(f)}{P_{\mathrm{F}}(f)}\right) .
\end{aligned}
$$


Those frequency-dependent functions can be compared with the amplitude and phase characteristics of the air-tight package signal related to the fully opened package in order to perform the fitting of the thermal wave model to the experimental data.

\subsection{Drum Effect in the Component's Package}

The model of the air-tight package can be extended by taking into account another phenomenon, which is the drum effect in the metal base of the component. In this case in the total reverse signal, the drum effect signal must be included. The latter can be described by

$$
\begin{aligned}
& P_{\text {drum }}(f)=\frac{I(f)}{\lambda_{2} \sigma_{2}^{2}(f)} \\
& \qquad\left(\frac{l_{2}}{2}-\frac{\exp \left(l_{2} \sigma_{2}(f)\right)+\exp \left(-l_{2} \sigma_{2}(f)\right)-2}{\sigma_{2}(f) \exp \left(l_{2} \sigma_{2}(f)\right)-\exp \left(-l_{2} \sigma_{2}(f)\right)}\right) \frac{1}{\cosh \left(l_{1} \sigma_{1}(f)\right)},
\end{aligned}
$$

where $\lambda_{2}$ is the thermal conductivity of the metal base.

The reverse-to-front signal ratio with the drum effect considered can be given by the formulae:

$$
\begin{aligned}
& A_{\mathrm{R} 2 \text { Fdrum }}(f)=\left|\frac{P_{\mathrm{R}}(f)+D P_{\text {drum }}(f)}{P_{\mathrm{F}}(f)}\right|, \\
& \varphi_{\mathrm{R} 2 \text { Fdrum }}(f)=\frac{180}{\pi} \arg \left(\frac{P_{\mathrm{R}}(f)+D P_{\text {drum }}(f)}{P_{\mathrm{F}}(f)}\right) .
\end{aligned}
$$

Here, $D$ is a coefficient representing the impact of the drum effect on the total reverse signal.

Figure 5 presents the curves of the presented air-tight package models together with the measurement points of a series of air-tight TO-39 packages related to a fully opened package signal. It can be seen that only in a narrow range of low frequencies the measurement data can be approximated with the thermal wave signal. For higher frequencies, the drum effect takes the leading role in the air-tight package signal and can be considered as the main limiting factor of the test method.

\section{Computation Procedure of Leakiness Contribution in the Measured Signal}

Depending on the dimension and the mechanical structure of the package, the background thermoacoustic signal can exceed the useful signal coming from the leakiness of a considerably large diameter. The useful leakiness signal can be extracted from the total measured signal as the result of a subtraction of vectors representing the signals in the frequency domain:

$$
\begin{aligned}
& A_{\mathrm{c}}=\left|A_{\mathrm{t}} \exp \left(\mathrm{j} \phi_{\mathrm{t}}(f)\right)-A_{\mathrm{p}} \exp \left(\mathrm{j} \phi_{\mathrm{p}}(f)\right)\right|, \\
& \phi_{\mathrm{c}}(f)=\frac{180}{\pi} \arg \left(A_{\mathrm{t}} \exp \left(\mathrm{j} \phi_{\mathrm{t}}(f)\right)-A_{\mathrm{p}} \exp \left(\mathrm{j} \phi_{\mathrm{p}}(f)\right)\right),
\end{aligned}
$$



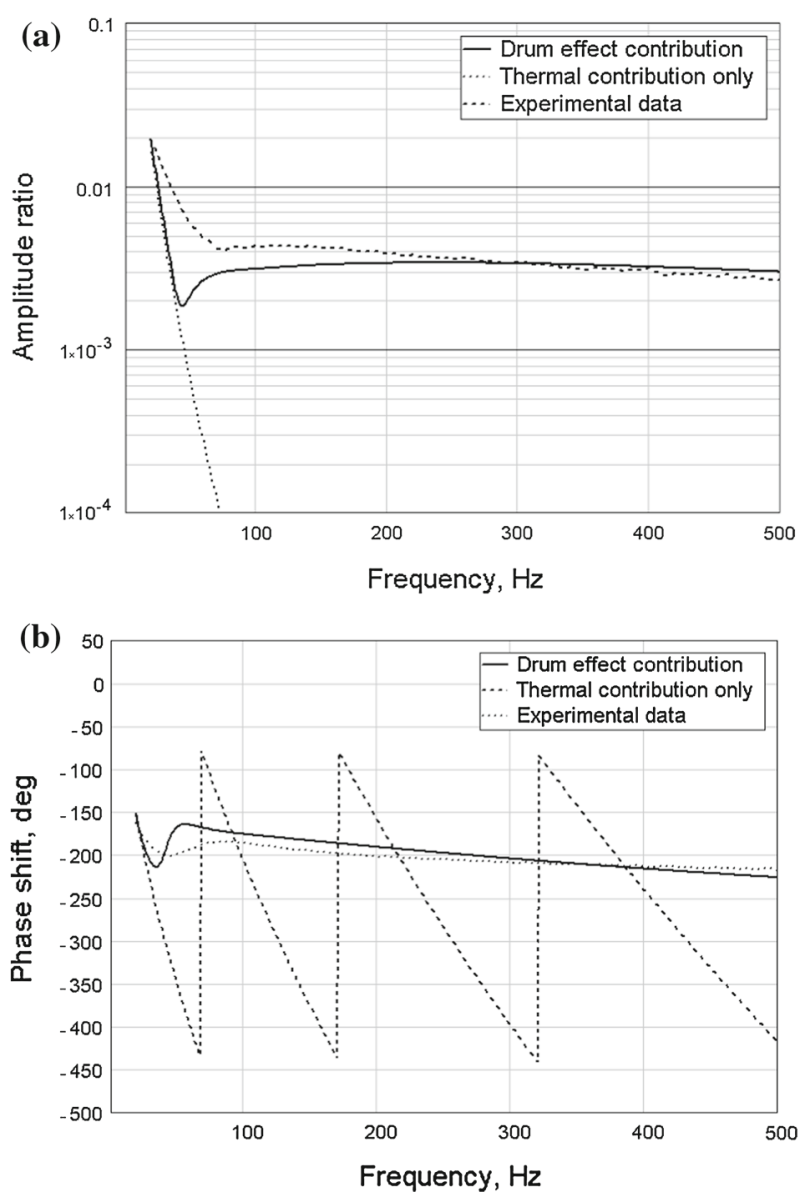

Fig. 5 Amplitude and phase frequency characteristics of air-tight TO-39 packaging

where $A_{\mathrm{t}}(f)$ and $\phi_{\mathrm{t}}(f)$ are total amplitudes and phases of the signal of the transistor with a leakiness, $A_{\mathrm{p}}(f)$ and $\phi_{\mathrm{p}}$ are amplitudes and phases of the air-tight transistor, and $A_{\mathrm{c}}(f)$ and $\phi_{\mathrm{c}}(f)$ are amplitudes and phases of the signal coming only from the leakiness.

The characteristics of an air-tight package for a given component type can be obtained statistically by the measurement of a series of air-tight packages. Figure 6 shows the results of the computation procedure performed for a TO-39 package with a particular leakiness.

\section{Conclusion}

Theoretical and experimental results confirmed that the CRLC model is appropriate for the modeling of thermoacoustic characteristics measured for the hermeticity test of transistors. Numerical computations proved that the drum effect of the packaging 

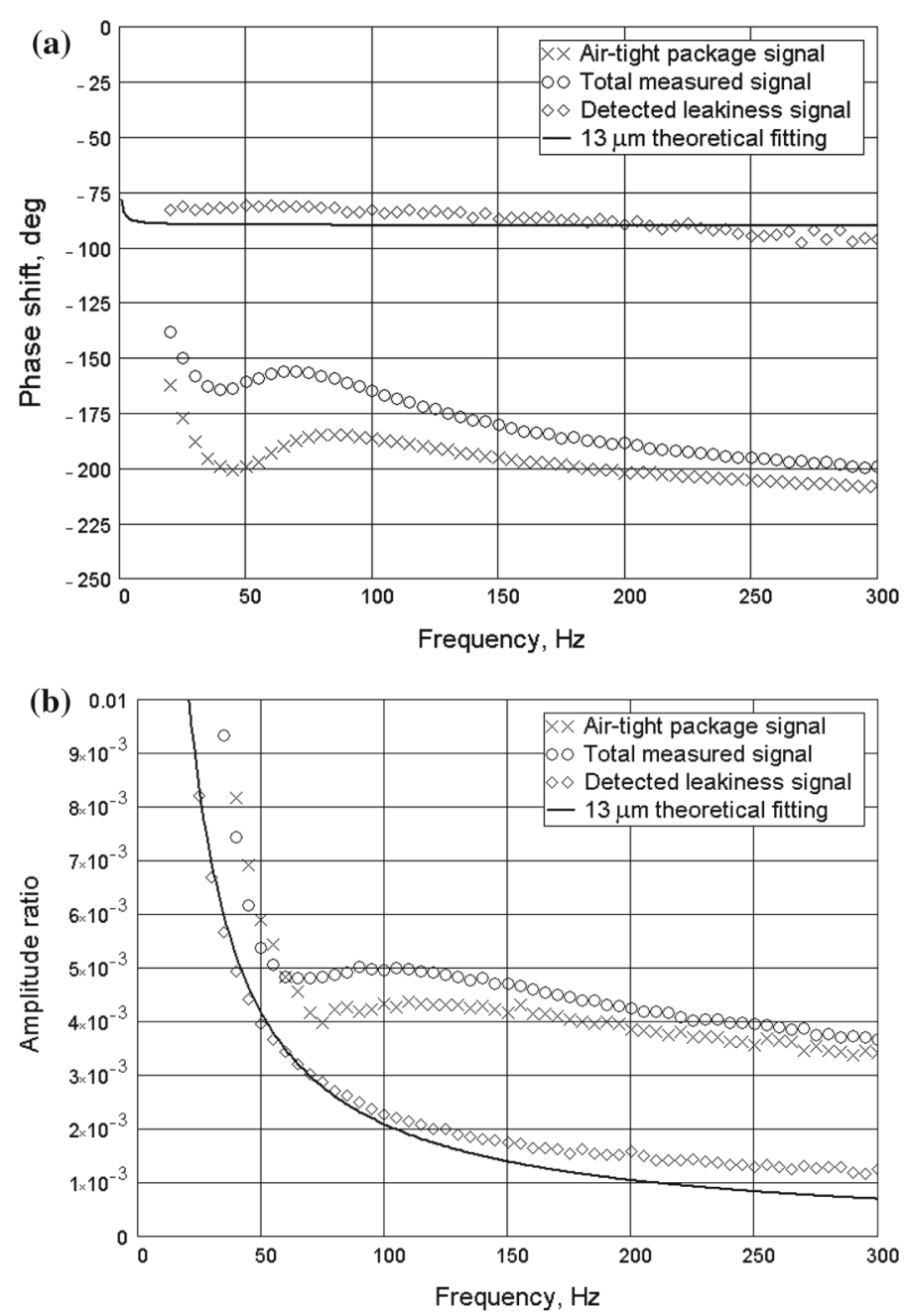

Fig. 6 Results of the leakiness size estimation for a TO-39 package

is the main limitation of this thermoacoustic method. The procedure of the computation of the hole contribution from the total thermoacoustic signal characteristic was presented and tested. It was proven that the thermoacoustic approach enables measurements and computations of the air-tightness of the packagings. The sensitivity of the method, depending on the size of the package was estimated to a hole radius of a few micrometers.

Open Access This article is distributed under the terms of the Creative Commons Attribution License which permits any use, distribution, and reproduction in any medium, provided the original author(s) and the source are credited. 


\section{References}

1. Military Standard MIL-STD-202G, Method 112E, Seal (U.S. Department of Defense, Washington, DC, 2003)

2. Military Standard MIL-STD-750D (Semiconductor Devices), Method 1071.6, Hermetic Seal (U.S. Department of Defense, Washington, DC, 1995)

3. Military Standard MIL-STD-883H (Microcircuits), Method 1014.13, Seal (U.S. Department of Defense, Washington, DC, 2010)

4. International Standard IEC 60068-2-17:1994, Environmental Testing-Part 2: Tests-Test Q: Sealing (International Electrotechnical Commission, Geneva, Switzerland, 1994)

5. JEDEC Standard, JESD22-A1-9-A, Hermeticity (JEDEC Solid State Technology Association, Arlington, VA, 2001)

6. D. Lellouchi, J. Dhennin, X. Lafontan, D. Veyrie, J.F. Le Neal, F. Pressecq, J. Micromech. Microeng. 20, 2 (2010)

7. D. Veyrie, J. Roux, F. Pressecq, A. Tetelin, C. Pellet, A new method to assess the hermeticity of MEMS micro-packages, in Proceedings of the 5th ESA Round Table on Micro/Nano Technologies for Space (Noordwijk, Netherlands, 2005)

8. S. Millar, M.P.Y. Desmulliez, S. McCracken, Leak detection methods for glass capped and polymer sealed MEMS packages, in 2010 Symposium on Design Test Integration and Packaging of MEMS/MOEMS (Seville, Spain, 2010)

9. F. Gueissaz, Ultra low leak detection method for MEMS devices, in 18th IEEE International Conference on Micro Electro Mechanical Systems (Miami, FL, 2005)

10. S. Millar, M.P.Y. Desmulliez, S. Cargill, S. McCracken, In-situ test structures for ultra low leak detection, in Electronic System-Integration Technology Conference (Edinburgh, UK, 2010)

11. S. Costello, M.P.Y. Desmulliez, S. McCracken, C. Lowrie, S. Cargill, A.J. Walton, Piezoresistive membrane deflection test structure for the evaluation of hermeticity in low cavity volume MEMS and microelectronic packages, in IEEE International Conference on Microelectronic Test Structures (Edinburgh, UK, 2012)

12. H. Grange, J.S. Danel, H. Feldis, B. Desloges, V. Jousseaume, C. Licitra, P.B. Manquat, P. Grosgeorges, B. Fassi, Z. Sbiaa, P. Robert, A new method for hermeticity measurements using porous ultra low $\mathrm{k}$ dielectrics for sub-ppm moisture detection, in Solid-State Sensors, Actuators and Microsystems Conference (Denver, CO, 2009)

13. F. Seigneur, T. Maeder, J. Jacot, Laser soldered packaging hermeticity measurement using metallic conductor resistance, in XXX International Conference of IMAPS (Poland Chapter, Kraków, Poland, 2005)

14. M. Maliński, Arch. Acoust. 30, 345 (2005)

15. M. Maliński, Acta Acust. United Ac. 91, 372 (2005)

16. L. Bychto, M. Maliński, Acta Acust. United Ac. 92, 482 (2006)

17. Ł. Chrobak, M. Maliński, Nondestruct. Test. Eval. 28, 17 (2013)

18. M. Maliński, in Fotoakustyka i spektroskopia fotoakustyczna materiatów pótprzewodnikowych (Koszalin University of Technology, Koszalin, Poland, 2004), pp. 70-79/262-369 